\title{
Bullous pemphigoid associated with the use of nivolumab in a patient with metastatic melanoma: a case report.
}

\author{
Penfigoide bolhoso associado ao uso de nivolumabe em paciente com melanoma \\ metastático: relato de caso.
}

Diego Lopes Paim Miranda', Leilah Andrade Alves' ${ }^{1}$ Dyonatas Rodrigues da Mata' ${ }^{1}$, Nathanael Pinheiro ${ }^{2}$, Anete Olivieri Pessoa da Silva ${ }^{3}$, Luciana Castro Garcia Landeiro ${ }^{4}$.

\begin{abstract}
Benefits of immunotherapy within modern oncology are evident, especially when evaluating survival, morbidity and systemic effects in the treatment of neoplastic tumors. However, adverse events have been described in association with checkpoint inhibitors. We aim to describe a case of bullous pemphigoid in a patient with metastatic melanoma treated with nivolumab. A 62-year-old male diagnosed with metastatic melanoma and BRAF mutation who underwent surgical resection started nivolumab and during the 40th cycle presented dermatological lesions compatible with bullous pemphigoid. After confirmation with biopsy, corticosteroid therapy was adopted and remission of the lesions was observed followed by relapse after immunotherapy reintroduction. Even though checkpoint inhibitors express several advantages over conventional systemic agents, these drugs seem to exert toxic effects that may manifest as skin lesions. Bullous pemphigoid diagnosis, which requires careful clinical investigation, should be considered when patients present dermatologic symptoms after treatment with nivolumab.
\end{abstract}

Keywords: Pemphigoid, Bullous; Nivolumab; Melanoma.

1. Universidade Federal da Bahia, Faculdade de Medicina - Salvador - Bahia - Brazil.

2. Imagepat Anatomia Patológica, Laboratório de Anatomia Patológica - Salvador - Bahia - Brazil.

3. Dermapp, Clínica de Dermatologia - Salvador - Bahia - Brazil.

4. Grupo Oncoclínicas, Núcleo de Oncologia da Bahia - Salvador - Bahia - Brazil.

Financial support: none to declare.

Conflicts of interest: The authors declare no conflict of interest relevant to this manuscript.

Correspondence author: Diego Lopes Paim Miranda.

E-mail: diegolpmiranda@hotmail.com 


\section{RESUMO}

Os benefícios da imunoterapia na oncologia moderna são evidentes, com vantagens significativas quanto à avaliação de sobrevida, morbidade e efeitos sistêmicos no tratamento de tumores neoplásicos. No entanto, eventos adversos têm sido associados ao uso de inibidores de checkpoint. Neste estudo, objetivou-se descrever um caso de penfigoide bolhoso em um paciente com melanoma metastático tratado com nivolumabe. Um homem de 62 anos com diagnóstico de melanoma metastático com mutação BRAF, submetido à ressecção cirúrgica, iniciou nivolumabe e durante $040^{\circ}$ ciclo, apresentou lesões dermatológicas compatíveis com penfigoide bolhoso. Após confirmação de biópsia, foi adotada terapia com corticosteróides e observada remissão das lesões, seguida de recidiva após reintrodução da imunoterapia. Embora os inibidores de checkpoint expressem várias vantagens sobre os agentes sistêmicos convencionais, tais medicamentos parecem exercer efeitos tóxicos que podem manifestar-se como lesões na pele. O diagnóstico de penfigoide bolhoso, o qual requer cuidadosa investigação clínica, deve ser considerado quando os pacientes apresentarem sintomas dermatológicos após tratamento com nivolumabe.

Descritores: Penfigoide bolhoso; Nivolumabe; Melanoma.

\section{INTRODUCTION}

Malignant melanoma is a type of skin cancer associated with high mortality rates and low response to chemotherapy or radiotherapy. Immunotherapy with checkpoint inhibitors has provided substantial survival gains along with lower toxic effects and better quality of life for patients with advanced disease. ${ }^{[1-3]}$ However, as these agents become broadly used, several uncommon immunerelated side effects are turning into new challenges for cancer therapeutics. ${ }^{[4,5]}$

Immunotherapy targeting immune checkpoints has changed the landscape of cancer treatment. Immune checkpoints, such as PD1, are inhibitory receptors expressed by $T$ lymphocytes ( $T$ cells). ${ }^{[6]}$ These receptors are activated by antigens derived from self-tissue, such as PD-L1, and subsequently transmit signals that limit excessive $T$ cell-mediated inflammatory responses. ${ }^{[7]}$ This is an important regulatory mechanism of immune homeostasis and also prevents autoimmunity. ${ }^{[7]}$ However, tumor cells have evolved to express immune checkpoint ligands which render tumor-specific $T$ cells inactive and therefore serve as a way of evading immune response. Checkpoint inhibitors, in turn, are monoclonal antibodies targeting immune checkpoints or their ligands.

By blocking these targets, unlike chemotherapy, that aims to directly destroy tumor cells, checkpoint inhibitors act indirectly by enhancing antitumor T cell response. ${ }^{[5]}$ These drugs have been shown to be effective against melanoma and other solid tumors, with dramatic improvements in disease outcome. [5] Nivolumab, a human IgG4 monoclonal antibody against programmed cell death protein 1 receptors (a-PD-1), has been clinically approved in many countries for the treatment of melanoma, metastatic non-small cell lung cancer, metastatic renal cell cancer and refractory Hodgkin's lymphoma. ${ }^{[8,9]}$

Despite improving treatment response and survival rates for several types of cancer, nivolumab is associated with a collection of immune reactions known as immune-related adverse events (irAE), such as thyroid dysfunction, hepatitis, pneumonitis, hypophysitis and colitis. ${ }^{[10]}$ Overall, dermatological toxicities such as exanthemas, lichenoid reactions, dermatitis, psoriasis, sarcoidosis, leukoderma, Steve-Johnson syndrome, and toxic epidermal necrolysis-like reactions are the most often related to immune checkpoint inhibitor therapies. [5,11] These manifestations are present in about 39\% and $17 \%$ of patients receiving a-PD-1 and a-PD-L1, respectively. ${ }^{[11]}$ Bullous dermatosis are less frequent adverse events, marked by blistering skin lesions that may involve significant body surface area, and potentially lead to treatment interruption or discontinuation. ${ }^{[5]}$ Bullous pemphigoid (BP), bullous lichenoid dermatitis or linear IgA bullous dermatosis have been reported in about $1 \%$ of patients treated with PD-1 or PD- L1 inhibitors. ${ }^{[5,9]}$

BP is a cutaneous autoimmune disorder that involves the expression of two autoantibodies against basement membrane zone (BMZ) autoantigens: BP180 (BPAG2) and BP230 (BPAG1). Both antigens are components of the hemidesmosome, which acts on 
adhesion between the dermis and the epidermis. The disease is characterized by immunoglobulins (IgG) and complement component C3 deposition in $\mathrm{BMZ}$, and manifests with pruritus, urticariform eruptions, vesicles, subepidermal blisters and, in rare cases, erosive mucosal lesions. ${ }^{[12-14]}$

Drug-induced BP most often appears in the first 6 to 8 months of treatment, with or without prodromes of nonspecific pruritus. ${ }^{[4,5]}$ The pathophysiology of drug-induced BP is the same as that believed to cause conventional BP, which causes clinical distinction to be difficult, and in both the majority of affected individuals are over 60-years-old. ${ }^{[4]}$ In most cases, the occurrence of BP requires immunotherapy discontinuation; [12] however, if the diagnosis is established early, immediate therapy with topical or systemic steroids can prevent the interruption of antineoplastic therapy. ${ }^{[12]}$

Diagnosis of BP relies on a combination of clinical criteria, immunopathologic examinations and serology. ${ }^{[4,9,12]}$ Histopathology demonstrates subepidermal blisters with inflammatory cell infiltrates containing eosinophils, neutrophils and lymphocytes, confirmed when both C3 complement and IgG antibody are detected on the epidermal basement membrane of the surrounding skin through direct immunofluorescence microscopy. $[4,9,12]$

Indirect immunofluorescence study detects IgG circulating autoantibodies that bind to the epidermal side of the skin basement membrane, on salt-split human skin substrate. It is also possible to detect specific circulating anti-basement membrane antibodies (a-BP180/BP230 and a-NC16A) in serum samples by enzyme-linked immunosorbent assay (ELISA). ${ }^{[4,9,12]}$ The first-line treatment is based on topical or systemic corticosteroids. ${ }^{[4,9]}$ Patients with generalized BP usually need systemic therapy. [4,9] Immunosuppressants such as azathioprine, mycophenolate mofetil or methotrexate can be added as glucocorticoid-sparing agents, but these agents may also cause serious adverse effects. ${ }^{[4]}$ Anti-inflammatory agents can be an option for mild cases, as well as biologic agents such a rituximab and omalizumab. ${ }^{[4,15]}$

In this report, we describe a case of PD-1 inhibitorinduced $\mathrm{BP}$ in a patient who received nivolumab as a third line treatment for metastatic melanoma. At first, a small blister appeared after 40 cycles of nivolumab, and evolved as several bullous lesions on his arms, back and abdomen. Immunopathologic examinations revealed the diagnosis of BP. Immunotherapy was suspended and full remission of the lesions was attained by treatment with prednisolone. However, after reintroduction of nivolumab, lesions returned with a diffuse pemphigoid-like pattern in multiple skin sites with local pain, indicating that BP emerged as an adverse event of immunotherapy.

\section{CASE REPORT}

A 62-year-old male patient was submitted to preoperatory tests for the resection of multiple nevus in his left auricular pavilion, in July of 2015, in which pulmonary nodules of secondary aspect were observed by chest X-ray. Afterwards, a biopsy guided by chest tomography of one pulmonary nodule was performed, with anatomopathological examination describing neoplastic cells with epithelioid morphology. Immunohistochemical analysis confirmed a malignant melanoma. PETCT indicated stage IV disease, with secondary involvement of lungs, pleura and left axillary lymph nodes. Genotyping of the BRAF gene showed presence of V600E mutation in exon 15.

The patient reported a previous diagnosis of malignant melanoma in 2007 on the left shoulder, with a Clark level II and with Breslow's thickness of
$0.4 \mathrm{~mm}$. He was submitted to resection (pathology report does not describe margins of the lesion) and the follow up was performed by a dermatologist. At the time, he was not referred for evaluation with an oncologist. As comorbidities, he reported diabetes mellitus and gout both diagnosed in 2000, and systemic hypertension diagnosed in 1995, in use of diuretics anti-diabetic drugs.

First-line chemotherapy started in August 2015, with dacarbazine $1000 \mathrm{mg} / \mathrm{m}^{2}$ on D1, every 03 weeks. Patient was treated for seven months, with partial response as best response, by RECIST.

In June 2016, the subject presented with hepatic, pulmonary and lymph node progression of metastasis, and was treated with vemurafenib 960mg daily as second line therapy. In the first evaluation of response to vemurafenib, in September 2016, PETCT showed exclusive progression of lymph node 
metastasis in the left axilla, associated with pain that limited mobility of the ipsilateral arm. Palliative radiotherapy was prescribed for better control of local pain, and the patient underwent conformational radiotherapy in the axilla, with a total dose of 3000cGy (300cGy for ten days), between September 21 and 30th, 2016. Shortly after radiotherapy, he started third-line treatment with nivolumab $3 \mathrm{mg} / \mathrm{kg}$, D1 every two weeks. The best response obtained was partial response (approximately 90\% reduction of the lesions).

Around the 40th cycle of nivolumab, in March 2018, the patient presented with a blister, smaller than $1 \mathrm{~cm}$ at its largest diameter, in the upper region of his right arm. In a follow-up visit two weeks later, the blister had evolved to an ulcerated healing lesion. One month later, it had changed to a flat, crusty lesion of $1.5 \mathrm{~cm}$ with a small satellite bullous lesion aside it, along with another small bullous lesion in the anterior region of the same arm, and a grade 1 rash on his face.

In May 2018, several bullous lesions appeared on his arms, back and abdomen, affecting less than $10 \%$ of his skin, none with signs of secondary infections and some with a crusty appearance, with pruritus and mild local discomfort. The bullae were tense, and there were no urticarial lesions or mucosal lesions associated. Pruritus was not a prominent symptom. Immunotherapy was suspended on suspicion of the bullous lesions being caused by nivolumab treatment.

The patient was referred for evaluation by a dermatologist, who biopsied one of the lesions. The histopathological examination on May 2018 reported bullous dermatosis with subepidermal cleavage and perivascular mononuclear infiltrate with eosinophils (Figures 1 and 2). Direct immunofluorescence confirmed compatibility with $\mathrm{BP}$ revealing strong (3+) linear staining for IgG and C3 along the epidermal basement membrane, in a " $\mathrm{N}$ " pattern, with no staining observed for IgA, IgM and C1q (Figures 3 and 4). Prednisolone 1mg/ $\mathrm{kg}$ was prescribed, with a complete resolution of the dermatological condition in 20 days. PET-CT indicated stable oncologic disease.

Subsequently, weaning of the corticoid was performed and nivolumab 3mg/kg, D1, every two weeks was resumed.

In the third cycle after reintroduction of nivolumab (August 2018), the patient presented again with a painless bullous lesion, on the back of his left hand. In October 2018, he complained of microblisters

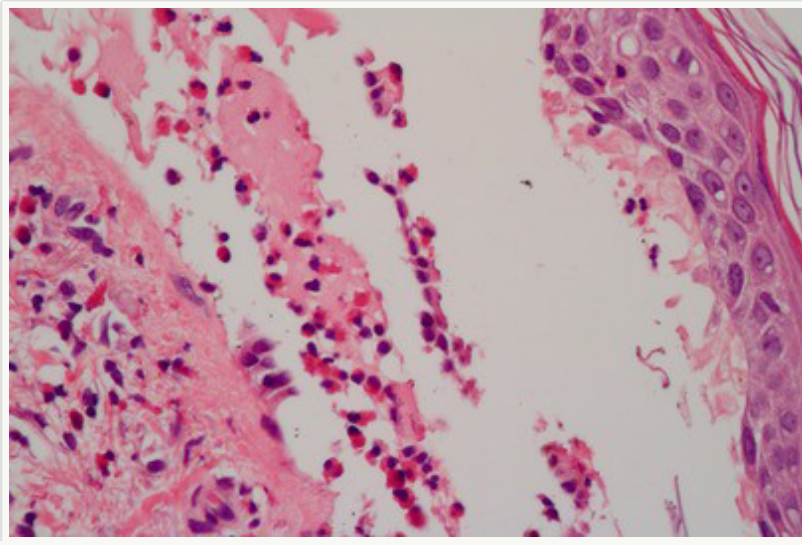

Figure 1. Subepidermal cleft with inflammatory infiltrate containing numerous eosinophils.

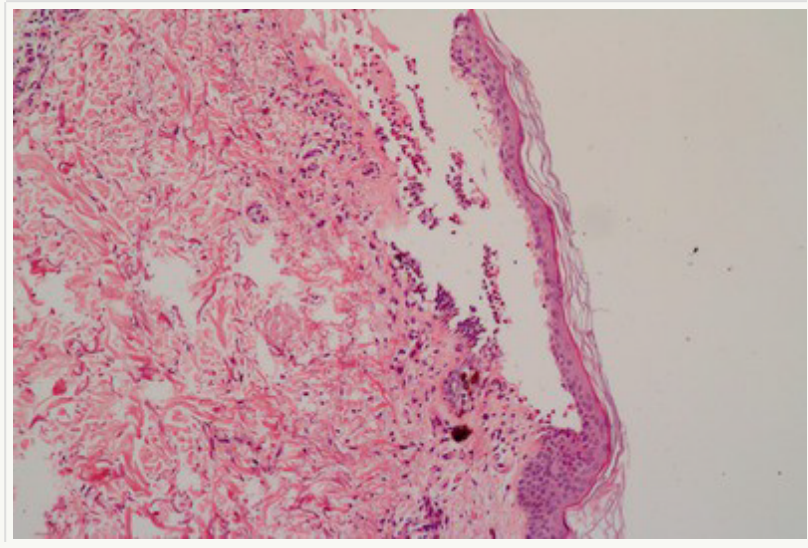

Figure 2. Subepidermal cleft with inflammatory infiltrate containing numerous eosinophils.

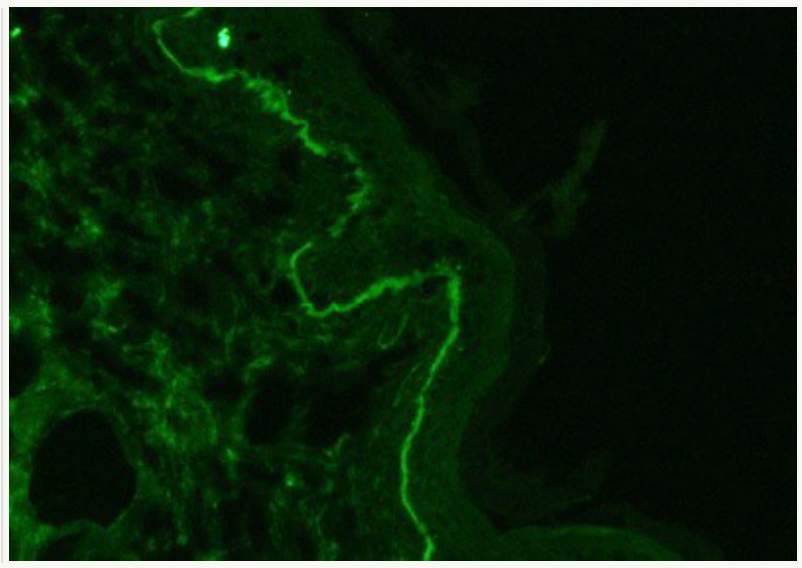

Figure 3. C3, linear in appearance, along the epithelium basement membrane.

spread on his back and upper left limb (grade 3), in addition to grade 1 fatigue. Once again, nivolumab was stopped due to diffuse pemphigoidlike lesions in multiple skin sites with local pain, and prednisolone $80 \mathrm{mg} /$ day was prescribed for 10 days. The weaning of the corticoid was initiated, but dose reduction to less than $10 \mathrm{mg} /$ day was associated with the onset of new painful bullous lesions, and the 


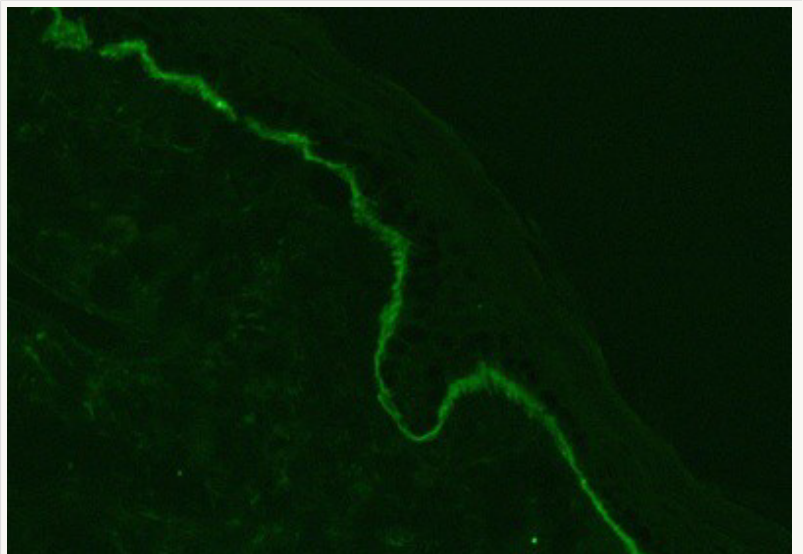

Figure 4. IgG, linear in appearance, along the epithelium basement membrane. patient was maintained on prednisolone 10mg/ day for approximately three months, when it was possible to progress to complete weaning.

After complete weaning of the corticoid, the patient was in clinical follow-up, without systemic treatment, with PET-CT of February 2019 showing stable disease. On April 03, 2019, patient presented with dysarthria and dizziness, and a single metastasis of $1.3 \mathrm{~cm}$ was diagnosed in precentral gyrus topography (skull MRI). He underwent radiosurgery on April 09, 2019, with a total dose of 2,100cGy. PET-CT of April 15, 2019, showed that, in addition to central nervous system involvement, he also presented metastatic disease progression to the lungs and bone (only one lesion). Then, on February 05, 2019, fourth-line treatment was initiated, with an oral combination of trametinib 2mg daily, plus dabrafenib 150mg BID, daily.

\section{DISCUSSION}

In the last few years, checkpoint inhibitors have replaced conventional chemotherapy as metastatic melanoma therapy, bringing numerous benefits in survival and quality of life. However, despite the approval of nivolumab as first-line therapy for the treatment of certain tumors, side effects associated with its administration, such as pneumonitis, thyroid dysfunction and hepatitis have often been described. $[4,5]$

Few cases of association between nivolumab monoclonal antibody and bullous pemphigoid have been reported on the literature. ${ }^{[12]}$ The diagnosis of BP should be made by clinical and laboratorial evaluation. It typically manifests with subepidermal tense blisters that can appear on any part of the skin and is more common in the elderly ( $>60$ years of age). ${ }^{[9]}$ Pruritus is frequently the first symptom of BP and it can precede blistering by weeks to months. [14] For this reason, immunological diagnostic studies for BP are warranted in patients treated with checkpoint inhibitors who develop intractable pruritus. However, in this case's patient, pruritus was not a prominent symptom.

The main differential diagnosis of bullous pemphigoid evidenced in the clinic are cicatricial pemphigoid, dermatitis herpetiformis, drug-induced bullous disorders, epidermolysis bullosa, epidermolysis bullosa acquisita, erythema multiforme and linear IgA dermatosis. ${ }^{[5]}$ In patients with clinical findings suggestive of bullous pemphigoid, such as this one, laboratory assessment should begin by obtaining both a lesion tissue specimen for routine (fixed tissue) histopathology and a perilesional tissue specimen for direct immunofluorescence (DIF) examinations. ${ }^{[4]}$ Detection of specific circulating IgG autoantibodies against basement membrane (a-B180/B230) in patient serum by ELISA can also be performed for diagnosis. ${ }^{[4]}$ Finally, BP can be confirmed by indirect immunofluorescence study, which detects IgG circulating autoantibodies that bind to the epidermal side of the skin basement membrane, on salt-split human skin substrate. ${ }^{[4]}$

In the anatomopathological examination of patient's biopsy, bullous dermatosis with eosinophils was observed in the infiltrate, confirmed with standard immunofluorescence compatible with bullous pemphigoid. Due to low volume, indirect immunofluorescence and salt split skin testing is not fully validated in our supporting Pathology Lab, and since the diagnosis of BP was confirmed by standard immunofluorescence, no further investigation was necessary. ${ }^{[4]}$ Subject was then treated with prednisolone $1 \mathrm{mg} / \mathrm{kg}$, with a complete resolution of the symptoms.

In this case report, signs of dermatological toxicity started to show after 40 cycles (9 months) of nivolumab, slightly later than expected for druginduced BP (typically 6 months) according to the available literature. ${ }^{[5]}$ Three differential diagnosis were acknowledged as possible underlying causes of BP: drug-induced BP secondary to nivolumab treatment; drug-induced BP associated to diuretics or 
anti-diabetic drugs; and BP as a rare paraneoplastic manifestation of melanoma.

Considering that the skin lesions started to show in parallel with introduction of nivolumab treatment, and that patient had been in use of all other medications for a long period previously to the rising of $\mathrm{BP}$, we find it unlikely that the disease could be related to diuretic and anti-diabetic drugs. In addition, after full recovery from the first BP manifestations, there was recurrence of the bullous lesions when nivolumab treatment resumed, which strongly suggests that this clinical condition emerged as a cutaneous immune-related adverse event induced by nivolumab and not as a rare paraneoplastic manifestation of melanoma. ${ }^{[13]}$ Therapy was then interrupted, and the patient was once again treated with prednisolone with complete resolution of dermatological features.

$\mathrm{BP}$ has a chronic course and is characterized by periods of exacerbation and remission, but in the absence of adequate treatment, it is progressive and strongly associated with a high morbidity and mortality. ${ }^{[9,16]}$ As possible treatments, topical or systemic steroids, methotrexate, prednisone and mycophenolate mofetil can be prescribed. $[13,17,18]$ Systemic corticoids are considered the current standard for BP treatment, especially for generalized disease. ${ }^{[9]}$ However, elderly patients should receive high-potency topical steroids instead of systemic corticosteroids. Treatments using systemic corticosteroids have been associated with significant side effects in this population, such as severe insulin-dependent diabetes mellitus and cardiovascular disorders.[19] Moreover, topical agents have been proven to reduce mortality in elderly people who require corticoid treatment for long periods, when compared to systemic agents. [19] In this case's patient, the choice of a systemic agent was supported mainly by our assessment regarding patient's adherence to treatment, which was considered by his family and caregivers to be less favorable if a topical agent was prescribed.

Finally, it is also extremely important to follow the protocol concerning time to suspend, time to stop and time to resume (when appropriate) treatment with nivolumab in the presence of skin rash. With manifestations of skin rash degrees 1 and 2 , it is possible to continue the treatment and to treat symptoms associated. Treatment should be suspended with the presence of a degree 3 cutaneous rash, and only resumed after grade 1 cutaneous lesions is achieved. In the occurrence of a grade 4 rash, nivolumab should not be resumed.

Bullous adverse events such as BP are associated with immunotherapy in $1 \%$ of patients and can lead to high morbidity and mortality. When generalized disease occurs, it may require systemic immunosuppressant drugs and often causes disruption of cancer treatment. Early detection of dermatological toxicity and autoimmune adverse events can reduce the need for systemic corticosteroids and the impact on cancer therapy, and immunological diagnostic studies are warranted in patients treated with checkpoint inhibitors who develop initial clinical symptoms, such as pruritus.

\section{ETHICS}

The adopted procedures were in accordance with the ethical standards and approved by the Research Ethics Committee of the Santo Antônio
Hospital (Salvador, Bahia, Brazil), with a CAAE 03588818.9.0000.0047. There were no Conflicts of Interest. The patient signed the informed consent approved by the Research Ethics Committee.

\section{ACKNOWLEDGMENTS}

We are grateful to Grupo Oncoclínicas, Núcleo de Oncologia da Bahia (NOB) and to the Liga Acadêmica de Oncologia (LAON, Faculdade de Medicina da UFBA). 


\section{AUTHOR'S CONTRIBUTION}

Diego Lopes Paim Miranda: Collection and assembly of data, Conception and design, Data analysis and interpretation, Manuscript writing.

Leilah Andrade Alves: Collection and assembly of data, Conception and design, Data analysis and interpretation, Manuscript writing.

Dyonatas Rodrigues da Mata: Collection and assembly of data, Conception and design, Data analysis and interpretation, Manuscript writing.
Nathanael Pinheiro: Data analysis and interpretation, Provision of study materials or patient.

Anete Olivieri Pessoa da Silva: Data analysis and interpretation.

Luciana Castro Garcia Landeiro: Collection and assembly of data, Conception and design, Final approval of manuscript, Manuscript writing, Provision of study materials or patient.

\section{REFERENCES}

1. Sosman JA. Overview of the management of advanced cutaneous melanoma. UpToDate [Internet]. 2019; [cited 2019 Aug 01]. Available from: https://www.uptodate.com/contents/overview-of-the-management-of-advanced-cutaneous-melanoma

2. Hodi FS, Chiarion-Sileni V, Gonzalez R, Grob J, Rutkowski P, Cowey $C L$, et al. Nivolumab plus ipilimumab or nivolumab alone versus ipilimumab alone in advanced melanoma (CheckMate 067): 4-year outcomes of a multicentre, randomised, phase 3 trial. Lancet Oncol. 2018 Nov;19(11):1480-92.

3. Schachter J, Ribas A, Long GV, Arance A, Grob JJ, Mortier L, al. Pembrolizumab versus ipilimumab for advanced melanoma: final overall survival results of a multicentre, randomised, open-label phase 3 study (KEYNOTE-006). Lancet. 2017 Oct;390(10105):1853-62.

4. Lopez AT, Geskin L. A case of nivolumab-induced bullous pemphigoid: review of dermatologic toxicity associated with programmed cell death protein-1/programmed death ligand-1 inhibitors and recommendations for diagnosis and management. Oncologist. 2018 Oct;23(10):111926.

5. Siegel J, Totonchy M, Damsky W, Berk-Krauss, Castiglione Junior F, Sznol M, et al. Bullous disorders associated with anti-PD-1 and antiPD-L1 therapy: A retrospective analysis evaluating the clinical and histopathologic features, frequency, and impact on cancer therapy. J Am Acad Dermatol. 2018 Dec;79(6):1081-8.

6. Haanen JBAG, Robert C. Immune checkpoint inhibitors. Prog Tumor Res. 2015;55-66.

7. Tocheva AS, Mor A. Checkpoint inhibitors: applications for autoimmunity. Curr Allergy Asthma Rep. 2017 Sep;17(10):72.

8. Seidel JA, Otsuka A, Kabashima K. Anti-PD-1 and anti-CTLA-4 therapies in cancer: mechanisms of action, efficacy, and limitations. Front Oncol. 2018 Mar;8:86.

9. Aggarwal P. Disproportionality analysis of bullous pemphigoid adverse events with PD-1 inhibitors in the FDA Adverse Event Reporting System. Expert Opin Drug Saf. 2019 Jul;18(7):62333.

10. Khair DO, Bax HJ, Mele S, Crescioli S, Pellizzari G, Khiabany $A$, et al. Combining immune checkpoint inhibitors: established and emerging targets and strategies to improve outcomes in melanoma. Front Immunol. 2019 Mar;10:453.

11. Curry JL, Tetzlaff MT, Nagarajan P, Drucker C, Diab A, Hymes SR, et al. Diverse types of dermatologic toxicities from immune checkpoint blockade therapy. J Cutan Pathol. 2017 Feb;44(2):158-76.

12. Anastasopoulou A, Papaxoinis G, Diamantopoulos P, Christodifou E, Benopoulou $\mathrm{O}$, Stratigos A, et al. Bullous pemphigoid-like skin lesions and overt eosinophilia in a patient with melanoma treated with nivolumab: case report and review of the literature. J Immunother. 2018 Apr;41(3):164-7.

13. Schadendorf D, Hodi FS, Robert C, Weber JS, Margolin K, Hamid $\mathrm{O}$, et al. Pooled analysis of long-term survival data from phase II and phase III trials of ipilimumab in unresectable or metastatic melanoma. J Clin Oncol. 2015 Jun;33(17):1889-94.

14. Lopez AT, Khanna T, Antonov N, Audrey-Bayan C, Geskin L. A review of bullous pemphigoid associated with PD-1 and PD-L1 inhibitors. Int J Dermatol. 2018 Jun;57(6):664-9.

15. Murrell DF, Ramirez-Quizon M. Management and prognosis of bullous pemphigoid. UpToDate [Internet]. 2019; [cited 2019 Aug 01]. Available from: https://www.uptodate.com/contents/management-and-prognosis-of-bullous-pemphigoid

16. Abdel-Wahab N, Shah M, Suarez-Almazor ME. Adverse events associated with immune checkpoint blockade in patients with cancer: a 
systematic review of case reports. PLoS ONE. $2016 \mathrm{Jul} ; 11(7): \mathrm{e} 0160221$.

17. Long H, Zhang G, Wang L, Liu Q. Eosinophilic skin diseases: a comprehensive review. Clin Rev Allergy Immunol. 2016 Apr;50(2):189-213.

18. Leiferman KM. Clinical features and diagnosis of bullous pemphigoid and mucous membrane pemphigoid. UpToDate [Internet]. 2019; [cited 2019 Aug 01]. Available from: https://www. uptodate.com/contents/clinical-features-anddiagnosis-of-bullous-pemphigoid-and-mucousmembrane-pemphigoid

19. Joly P, Roujeau JC, Benichou J, Delaporte E, D'Incan M, Dreno B, et al. A comparison of two regimens of topical corticosteroids in the treatment of patients with bullous pemphigoid: a multicenter randomized study. J Invest Dermatol. 2009 Jul;129(7):1681-7. 